\title{
A Generalized Edge of the Wedge Theorem
}

\author{
$\AA$. Kolin and B. NAGEL \\ Department of Theoretical Physics, Royal Institute of Technology, Stockholm
}

Received September 1, 1967

\begin{abstract}
The edge of the wedge theorem is generalized to the case where one only assumes the existence and equality of the distribution boundary values of $f_{ \pm}(z)$ and all their derivatives on some analytic curve $\mathscr{C}$ in $R^{n}$. Here $f_{ \pm}(z)$ are holomorphic in $R^{n} \pm i C$, respectively, where $C$ is a convex cone, and $\mathscr{C}$ has its tangent vector in $C$ at every point. Under these assumptions there exists an analytic continuation $f(z)$ holomorphic in some complex neighbourhood of the double cone generated by $\mathscr{C}$. A proof is also given of the connection between the existence of a distribution boundary value and the growth of the holomorphic function near the boundary.
\end{abstract}

\section{Introduction}

The edge of the wedge theorem is an often used tool in quantum field theory (see [1], Ch. 2-5, and [2], §27, for proof, discussion, and references). This theorem states that given two functions $f_{ \pm}(z)$ of $n$ complex variables $z=\left(z_{1}, \ldots, z_{n}\right)$, holomorphic in the two tubes $R^{n} \pm i C$, respectively ( $C$ is an open convex cone with vertex in the origin), and having equal boundary values in the distribution sense on some open set $\Omega$ in $R^{n}$, there is a common analytic continuation $f(z)$ of $f_{ \pm}(z)$, holomorphic in some complex neighbourhood of $\Omega$. (We are not concerned here with the case studied by Epstein [3], where one has two cones $C_{1}$ and $C_{2}$ with $C_{1} \cap\left(-C_{2}\right)=\emptyset$.) As derivation is a continuous operation on distributions, existence and equality of the boundary values of $f_{ \pm}(z)$ on $\Omega$ imply the existence and equality of the boundary values of all pairs of derivatives $f_{ \pm}^{(p)}(z)$ on $\Omega$.

An extension of the edge of the wedge theorem in the case $n \geqq 2$ is the theorem of the $C$-convex hull ([2], $\S 28 ;[4])$, stating that under the assumptions above the holomorphy domain of $f(z)$ can be enlarged to contain a complex neighbourhood of the $C$-convex hull $B_{C}(\Omega)$ of $\Omega$. Here $B_{C}(\Omega)$ is the smallest set containing $\Omega$, such that if the segment $\left[x, x^{\prime}\right]$ belongs to $B_{C}(\Omega)$ then so does the double cone $(x+C) \cap\left(x^{\prime}-C\right)$. This result is proved with analytic completion techniques (continuity theorem).

It might be of interest to ask what conclusions can be drawn if we assume only the equality of the boundary values of $f_{ \pm}(z)$ and all derivatives on some lower-dimensional subset of $\Omega$. The first difficulty is what

13 Commun. math. Phys., Vol. 8 
we shall mean by the boundary value on this subset, as a distribution can in general not be given a meaning except on open subsets of the same dimension as $\Omega$. It is a natural requirement that the existence of a boundary value on $\Omega$ should imply the existence of a boundary value on the lower-dimensional subset in question.

To avoid this difficulty for the moment we assume the boundary values on $\Omega$ to exist as infinitely differentiable $\left(C^{\infty}\right)$ functions, uniformly approached as we go to the real space. It follows from the edge of the wedge theorem that if the boundary value of $f_{+}(z)$ on $\Omega$ is zero, then $f_{+}(z) \equiv 0$. But although it is trivial that if $f(z)$ and all derivatives are zero (we shall then say that $f(z)$ has an infinite zero or vanishes of infinite order) in some interior point of the holomorphy domain, then $f(z) \equiv 0$, one can give examples of non-zero functions having an infinite zero in every point of an (n-1)-dimensional submanifold of the boundary. In the case $n=1$ we can choose ([5], p. 320)

$$
f(z)=\int_{0}^{\infty} e^{i z k} e^{-k^{\mu} \cos \mu \pi} \sin \left(k^{\mu} \sin \mu \pi\right) d k, \quad 0<\mu<\frac{1}{2} .
$$

This function is holomorphic in $\operatorname{Im} z>0$ and has a $C^{\infty}$ boundary value on $\operatorname{Im} z=0$. This boundary function has an infinite zero in the point $\operatorname{Re} z=0$, as

$$
\int_{0}^{\infty} k^{N} e^{-k^{\mu} \cos \mu \pi} \sin \left(k^{\mu} \sin \mu \pi\right) d k=0, \quad N=0,1, \ldots
$$

If we define $g\left(z_{1}, \ldots, z_{n}\right)=f\left(z_{1}\right) \ldots f\left(z_{n}\right)$ we get a non-zero function, holomorphic in the product of the upper half planes, having a $C^{\infty}$ boundary value on $R^{n}+i\{0\}$, and vanishing of infinite order on all (n-1)-dimensional coordinate planes in $R^{n}$.

It can be noted that an application of the construction used in the theorem of the $C$-convex hull would here not give any extension of the original domain of infinite zeros. This remark gives a hint to what property the relevant subset of $\Omega$ should have to make possible a generalization of the edge of the wedge theorem: by forming the $C$-convex hull we should arrive at an open subset of $\Omega$. It turns out that this is actually the case and that it is enough to work with one-dimensional submanifolds in $R^{n}$. We define a $C$-like analytic curve $\mathscr{C}$ in $\Omega$ to be a curve $\{x(t) ; 0<t<1\}$ in $\Omega$, where all $x_{i}(t)$ are real-analytic and the tangent vector $x^{\prime}(t)$ lies in $C$ for every $t$.

Assume that $f_{ \pm}(z)$, holomorphic in $R^{n} \pm i C$, have together with all derivatives boundary values as $y \rightarrow 0$ in $\pm C$ on some $C$-like analytic curve $\mathscr{C}$, and that these boundary values are equal in pairs (in short: the boundary values on $\mathscr{C}$ exist and are equal to infinite order). The main theorem of this paper then states that there is a common analytic con- 
tinuation $f(z)$ of $f_{ \pm}(z)$, holomorphic in some complex neighbourhood of the double cone $(x(0)+C) \cap(x(1)-C)$ generated by $\mathscr{C}$ in $R^{n}$. See (II. 10) for the precise general form of the theorem.

The boundary value on $\mathscr{C}$ is here assumed to exist as a one-dimensional distribution in the sense defined in (II. 5). Especially, if the boundary value exists in the distribution sense on some open set containing $\mathscr{C}$ then the boundary values of infinite order on $\mathscr{C}$ also exist and are, in fact, infinitely differentiable under rigid translations of $\mathscr{C}$. The generalized edge of the wedge theorem proved here thus contains both the ordinary edge of the wedge theorem and the theorem of the $C$-convex hull. It is remarkable that this generalized theorem can be proved using only the same Cauchy representation formula that is used in the proof of the ordinary edge of the wedge theorem exhibited in [1].

An important role in the developments here is played by the criterion for the existence of distribution boundary value of a holomorphic function, stating roughly that the function should not increase faster than some inverse power of the distance to the boundary in $R^{n}$. Although this criterion seems to be common knowledge among those working in the field, we have not found any published proof ${ }^{1}$. We therefore include a proof for a slightly more general case (III. 2, Th. 1).

As we remarked above, if the boundary value of $f(z)$ on $R^{n}$ exists then also the boundary value on every $C$-like analytic curve exists and is $C^{\infty}$. This implies that we can define the boundary value on a $m$-dimensional real-analytic submanifold $(m<n)$ of $R^{n}$, provided every point of it belongs to some $C$-like curve lying in the manifold. This $m$-dimensional distribution boundary value is infinitely differentiable under rigid transJations of the manifold in $R^{n}$. Applying this result to the vacuum expectation value $\left(\Phi_{0}, A\left(x_{1}\right) \ldots A\left(x_{n}\right) \Phi_{0}\right)$, where $x_{i}$ are four-vectors and $A(x)$ is a quantized field satisfying the spectral condition, one finds that it makes sense to average in each $x_{i}$ over some time-like analytic curve $\mathscr{C}_{i}$. As a consequence averageing the field $A(x)$ over a time-like analytic curve gives a welldefined operator in Hilbert space, which is infinitely differentiable under translations of the curve. This is a natural generalization of Borchers' result [6].

The original motivation for the investigation reported here came from a paper by Greenberg [10] on the support properties of a field in momentum space. In case $\mathscr{A}$ of this paper it is shown that if the Fourier transform $\widetilde{A}(p)$ of a local field has an infinite zero on a hyperboloid $p^{2}=p_{0}^{2}$,

1 The reference in ([6], Lemma 10) to a paper by TiLLmann [7] is, we think, inadequate for several reasons, the first one being the fact that [7] only deals with distributions with compact support. In a later paper [8] Tillnians has given a representation formula for a general distribution in $R^{1}$ but the extension to $R^{n}$, $n \geqq 2$, is probably more difficult (cf. remark in [9], end of 13.8). 
where $p_{0}$ is a space-like vector, then the field is a generalized free field. To give a meaning to the vanishing of infinite order of $\widetilde{A}(p)$, and also in the continuation of the proof to increase the region of vanishing, Greenberg uses the Garrding representation of a causal distribution and known properties of distribution valued solutions of the wave equation (see e.g. [11]). These properties are that averaging over a time interval (or, by Lorentz invariance, over some time-like straight segment) gives a $C^{\infty}$ function in the space variables, and that a zero of infinite order on a time interval of a solution implies the vanishing of the solution in the corresponding double cone. Although it seems reasonable to assume that the same conclusions should be valid if we take a time-like analytic curve - which is what is needed in GREENBERG's proof - instead of a time-like line segment, it is not obvious how the original proofs should be modified to cover this case. The results of the present paper provide an independent proof for case $\mathscr{A}$ in [10], without using the Garding representation.

Alternatively, it follows from this paper that any solution $u \in \mathscr{S}^{\prime}$, the space of tempered distributions, of the wave equation (or the KleinGordon equation) has the two properties referred to above even relative to some arbitrary time-like analytic curve. In fact, the Fourier transform of $u$ has its support in the union of the forward and backward closed light cones, and $u$ can then be written as a difference between the boundary values of two functions holomorphic in the forward and backward tubes, respectively.

The rest of the paper is divided into two parts. In II the necessary notations are introduced and the general chain of ideas leading up to the main theorem is given without proofs, for the benefit of the reader who does not want to go too deeply into the sometimes rather lengthy proofs, which are found in III.

\section{Notations, Survey of Preliminary Results, and Statement of the Main Theorem}

1. The symbols $\Omega$ and $\Sigma \subset \Omega$ denote open connected sets in $R^{n}$. $\mathscr{D}(\Omega)$ is the space of infinitely differentiable functions with compact support in $\Omega$, and $\mathscr{D}^{\prime}(\Omega)$ is its dual, the space of distributions in $\Omega$. $C$ is an open convex cone in $R^{n}$ with vertex in the origin. A compact subcone $\bar{C}^{\prime}$ of $C$ is a closed cone, vertex $\{0\}$ included, such that its intersection with the unit sphere $\left\{y=\left(y_{1}, \ldots, y_{n}\right) ;|y|=\left(\Sigma y_{i}^{2}\right)^{1 / 2}=1\right\}$ in $R^{n}$ is a compact subset of the intersection of $C$ with the unit sphere. $C_{\delta}=C \cap\{y ;|y|<\delta\}$. In the case $n=1$ we assume $C=\{y ; y>0\}$, and then of course $\bar{C}^{\prime}=\{y ; y \geqq 0\}$. 
$O_{C}(\Omega)$ is an open connected set in $C^{n}$ of the form $O \cap\left(R^{n}+i C\right)$ where $O$ is an open neighbourhood of $\Omega$ in $C^{n}$. $O_{-C}(\Omega)=O \cap\left(R^{n}-i C\right)$. Evidently, for every compact subset $K$ of $\Omega$ we can find a $\delta>0$ such that $K+i C_{\delta} \subset O_{C}(\Omega), K-i C_{\delta} \subset O_{-C}(\Omega)$.

2. Assume $f(z)$ is a function defined and holomorphic in $O_{C}(\Omega)$. If $\varphi \in \mathscr{D}(\Omega)$, then supp $\varphi \subset K$ where $K$ is some compact set in $\Omega$, and with $\delta$ chosen as above

$$
\begin{aligned}
F_{y}(\varphi) & =\int f(z) \varphi(x) d x \\
& =\int f\left(x_{1}+i y_{1}, \ldots, x_{n}+i y_{n}\right) \varphi\left(x_{1}, \ldots, x_{n}\right) d x_{1} \ldots d x_{n}
\end{aligned}
$$

is defined and continuous for $y \in C_{\delta}$. It may happen that $\lim F_{y}(\varphi)$, $y \rightarrow 0$ in $C_{\delta}$, exists in the sense that $F_{y}(\varphi), y \in C_{\delta}$, and the limit value $F_{0}(\varphi)$ together give a function continuous in every $\bar{C}_{\delta}^{\prime}$. This means that the limit $F_{0}(\varphi)$ is approached uniformly in direction in every $\bar{C}^{\prime}$.

If the limit exists in the above sense for every $\varphi \in \mathscr{D}(\Omega)$ it defines an element $F=F_{0} \in \mathscr{D}^{\prime}(\Omega)$, the boundary value of $f(z)$ on $\Omega$. (See III. 1.)

A necessary and sufficient condition for $f(z)$ to have a boundary value on $\Omega$ is (III. 3 , Cor. 1 ):

For every compact $K \subset \Omega$ and compact subcone $\bar{C}^{\prime} \subset C$ we can find $\delta>0, M>0$, and non-negative integer $m$ such that

$$
|f(z)| \leqq M|y|^{-m}, \quad z \in K+i\left(\bar{C}_{\delta}^{\prime}-\{0\}\right) .
$$

Here $\delta$ may depend on $K, M$ and $m$ on $K$ and $\bar{C}^{\prime}$.

3. The ordinary edge of the wedge theorem states that given a pair of functions $f_{ \pm}(z)$ holomorphic in $O_{ \pm C}(\Omega)$, respectively, and having equal boundary values on $\Omega$, there exists a common analytic continuation $f(z)$ of $f_{+}(z)$, holomorphic in some complex neighbourhood of $\Omega$.

As derivation is a continuous operation in $\mathscr{D}^{\prime}(\Omega)$, existence and equality of boundary values of $f_{ \pm}(z)$ imply the existence and equality of boundary values of any pair of derivatives $f_{ \pm}^{(p)}(z)$.

In the generalized form of the theorem that we are going to prove one only assumes the existence and equality of boundary values of $f_{ \pm}(z)$ and all pairs of derivatives on some special curve $\mathscr{C}$ in $\Omega$. From this we infer the existence of an analytic continuation holomorphic in some complex neighbourhood of an open set $B(\mathscr{C}, \Omega, C)$ (II. 9) containing $\mathscr{C}$ in $\Omega$.

4. A $C$-like analytic curve $\mathscr{C}$ in $\Omega$ is a mapping of the open unit interval $I=(0,1)$ into $\Omega: t \rightarrow \alpha(t)=\left(\alpha_{1}(t), \ldots, \alpha_{n}(t)\right)$, where all $\alpha_{i}(t)$ are real-valued analytic functions in $I$, and every tangent vector $\alpha^{\prime}(t) \in C$, $t \in I$. We can extend this mapping to a holomorphic mapping $\alpha(\tau)$, $\tau=t+i s$, of some neighbourhood $J$ of $I$ in $C^{1}$ into $C^{n}$. Given $O_{ \pm C}(\Omega)$, $J$ can be chosen so small that the images of $J \cap\{\tau ; s \gtrless 0\}$ are contained in $O_{ \pm C}(\Omega)$. 
5. We shall now define what we mean by the statement that a function $f(z)$ holomorphic in $O_{C}(\Omega)$ has a boundary value, an element in $\mathscr{D}^{\prime}(I)$, on the $C$-like analytic curve $\mathscr{C}$ in $\Omega$.

Given $\phi \in \mathscr{D}(I)$, it is evident that for $s$ small positive, say $0<s \leqq \delta^{\prime}$, we can find $\delta$ and $\varepsilon$ such that for an arbitrary subcone $\bar{C}^{\prime}$ of $C$ and arbitrary $k>0$

$$
F_{s, \zeta}(\varphi)=\int f(\alpha(t+i s)+\zeta) \varphi(t) d t
$$

is a continuous function of $s$ and $\zeta$ when $0<s \leqq \delta^{\prime}$ and $\zeta$ belongs to the compact set $A\left(\bar{C}_{\delta}^{\prime}, \varepsilon, k\right)=$ the closure of

$$
\left\{\zeta=\xi+i \eta ; \eta \in \bar{C}_{\delta}^{\prime},|\xi|<\min (\varepsilon, k|\eta|)\right\} \text {. }
$$

It may happen that for every $\bar{C}^{\prime}$ the limit value as $s \rightarrow 0$ of this function exists, uniformly when $\zeta$ varies in $A\left(\bar{C}_{\delta}^{\prime}, \varepsilon, k\right)$, for some $k=k\left(\bar{C}^{\prime}\right)>0$. We thus get a function $F_{s, \zeta}(\varphi)$, continuous in the compact set $0 \leqq s \leqq \delta^{\prime}, \zeta \in A\left(\bar{C}_{\delta}^{\prime}, \varepsilon, k\right)$, for every $\bar{C}^{\prime}$.

If this is true for every $\varphi \in \mathscr{D}(I)\left(\delta^{\prime}, \delta\right.$, and $\varepsilon$ depend on $\operatorname{supp} \varphi$, in general) the limit value corresponding to $\zeta=0$ is easily seen to define an element $F_{\mathscr{C}}=F_{0,0}$ in $\mathscr{D}^{\prime}(I)$, which is called the boundary value of $f(z)$ on $\mathscr{C}$.

As in (II. 2) it is possible to give a necessary and sufficient condition on $f(z)$ for the boundary value on $\mathscr{C}$ to exist (III. 3, Cor. 2):

For every compact $K \subset \mathrm{I}$, every $\bar{C}^{\prime}$, appropriately chosen $\delta^{\prime}, \delta$, and $\varepsilon$, and some $k=k\left(\bar{C}^{\prime}\right)>0$ we can find $M>0$ and a non-negative integer $m$, such that

$$
|f(\alpha(\tau)+\zeta)| \leqq M s^{-m}, \quad(\tau, \zeta) \in\left[K+i\left(0, \delta^{\prime}\right)\right] \times A\left(\bar{C}_{\delta}^{\prime}, \varepsilon, k\right) .
$$

If the boundary values on $\mathscr{C}$ exist for $f(z)$ and all derivatives $f^{(p)}(z)$, we shall say that $f(z)$ has boundary values of infinite order on $\mathscr{C}$. For this to be the case we must of course have a majoration of the above type for $f(z)$ and each derivative.

6. Using the criteria in (II.2) and (II.5) for the existence of boundary values it easily follows that if $f(z)$ has a boundary value on some open real neighbourhood $\Sigma$ of $\mathscr{C}$, then $f(z)$ has boundary values of infinite order on $\mathscr{C}$. If the distance between the boundary of $\Sigma$ and $\mathscr{C}$ is $d>0$ the limit distribution on $\mathscr{C}$ as $s \rightarrow 0, \eta \rightarrow 0$, exists even without the assumption $|\xi|<k|\eta|$; we only need to assume $|\xi|<d$ and we then get a distribution $F_{\xi}$ which as element in $\mathscr{D}^{\prime}(I)$ is infinitely differentiable in $\xi$ for $|\xi|<d$. ("The boundary value on $\mathscr{C}$ is infinitely smooth under rigid translations of the curve $\mathscr{C}$ '.) The derivatives of $F_{\xi}$ are the boundary values of the corresponding derivatives of $f(z)$ (III. 3, Cor. 3).

That the assumption of existence of boundary values of infinite order on $\mathscr{C}$ is a weaker condition on $f(z)$ than the existence of boundary value on an open set containing $\Omega$ can be seen from the function $\exp (i v / z)$. 
Here $z$ and $w$ are one-dimensional variables, $\Omega=R^{2}, C=\{(y, v) ; y>0$, $v>0\}, \mathscr{C}=\left\{\left(-\frac{1}{2}+t,-\frac{1}{2}+t\right)\right\}$. Application of the criteria in (II. 2) and (II. 5) shows that this function has boundary values of infinite order on $\mathscr{C}$ but not on any open set containing the origin.

7. The function $f(z)$ in $O_{C}(\Omega)$ is uniquely determined by its boundary values of infinite order on $\mathscr{C}$ (III. 6 , Th. 2). To get this uniqueness it is actually enough to assume the existence of the limit distributions when we approach the real space along the complex continuation $\alpha(\tau)$ of $\mathscr{C}$, i.e. we assume $\lim \int f(\alpha(t+i s)) \varphi(t) d t, s \rightarrow 0$, to exist for all $p \in \mathscr{D}(I)$ and similarly for all derivatives $f^{(p)}(z)$. The example $\exp \left(i[w-z] / z^{2}\right)$, $\Omega, C$, and $\mathscr{C}$ as in (II. 6), shows that this is a weaker condition than existence of boundary values on $\mathscr{C}$.

8. The preceding point implies that if we have two functions $f_{1}(z)$ and $f_{2}(z)$ in $O_{C}(\Omega)$, or both in $O_{-C}(\Omega)$, which are equal to infinite order on $\mathscr{C}$, then $f_{1}=f_{2}$.

The main theorem we are going to formulate shows what happens if we have two functions $f_{ \pm}(z)$ holomorphic in $O_{ \pm C}(\Omega)$, respectively, which agree to infinite order on $\mathscr{C}$.

The equality to infinite order of the boundary values on $\mathscr{C}$ especially follows if the boundary values of $f_{+}(z)$ exist and are equal on some neighbourhood $\Sigma$ of $\mathscr{C}$ (III. 3, Cor. 4).

As existence of boundary values on $\Sigma$ implies the existence of boundary values to infinite order on $\mathscr{C}$ (II. 6 ) it is in the case that the boundary values of $f_{ \pm}(z)$ on $\Sigma$ are known to exist enough to check that the limit distributions on $\mathscr{C}$, defined in the sense given in (II. 7), are equal to infinite order.

9. With every $C$-like analytic curve $\mathscr{C}$ in $\Omega$ we associate the $C$-convex hull $B(\mathscr{C}, \Omega, C)$ of $\mathscr{C}$ in $\Omega$. This is the smallest open set in $\Omega$ such that

a. $B$ contains $\mathscr{C}$, and

b. if $x$ and $x^{\prime}$ are points in $B$ such that the set

$$
S_{x x^{\prime}}=(x+C) \cap\left(x^{\prime}-C\right) \subset \Omega, \quad \text { then also } S_{x x^{\prime}} \subset B .
$$

Thus, if the double cone $\lim _{\varepsilon \rightarrow 0}(\alpha(\varepsilon)+C) \cap(\alpha(1-\varepsilon)-C)$ is contained in $\Omega$, then $B$ is just this set.

10. We can now state the main theorem:

Generalized Edge of the Wedge Theorem. $f_{ \pm}(z)$ are functions holomorphic in $O_{ \pm C}(\Omega)$, respectively. The boundary values of these functions exist to infinite order on some $C$-like analytic curve $\mathscr{C}$ in $\Omega$.

If these boundary values are equal there is a common analytic continuation $f(z)$ of $f_{ \pm}(z)$, holomorphic in some complex neighbourhood of $B(\mathscr{C}, \Omega, C)$. This neighbourhood depends on $O_{{ }_{C}}(\Omega)$ and $B(\mathscr{C}, \Omega, C)$ but not on the functions $f_{ \pm}(z)$. 


\section{Auxiliary Theorems and Proof of the Main Theorem}

1. We first want to show that the functional on $\mathscr{D}(\Omega)$ defined according to (II. 2$)$ by $F(\varphi)=\lim \int f(z) \varphi(x) d x, y \rightarrow 0$, actually belongs to $\mathscr{D}^{\prime}(\Omega)$. It is evident that $F$ is linear; it remains to be shown that it is continuous, or, what is the same thing ([12], Ch. III, Th. V) that it is bounded on bounded sets in $\mathscr{D}(\Omega)$. If $B$ is a bounded set in $\mathscr{D}(\Omega)$ it is also a bounded set in some $\mathscr{D}\left(\Omega_{K}\right)$, where the closure of $\Omega_{K}$ is compact in $\Omega$ ([12], Ch. III, Th. IV). But for functions in $\mathscr{D}\left(\Omega_{K}\right) f(z)$ defines a distribution for all $y$ in some $C_{\delta}$, so $F$ restricted to $\mathscr{D}\left(\Omega_{K}\right)$ is the limit as $y \rightarrow 0$ of a sequence of distributions in $\Omega_{K}$. Hence $F \in \mathscr{D}^{\prime}\left(\Omega_{K}\right)$ ([12], Ch. III, Th. XIII) and is thus bounded on $B$.

2. We shall now study the connection between the existence of the boundary value $F$ or $F_{\mathscr{C}}$ and the growth of the function $f(z)$ as $z$ approaches the boundary.

We start with

Theorem 1. Consider a function $f(z, \zeta)$ defined and continuous for $(z, \zeta) \in O_{C}(\Omega) \times \mathscr{K}$ where $\mathscr{K}$ is a compact set in some topological space. For every $\zeta \in \mathscr{K} f(z, \zeta)$ is holomorphic as function of $z$ in $O_{C}(\Omega)$.

Given $\varphi \in \mathscr{D}(\Omega)$ we can find a $\delta>0$ such that $F_{y, \zeta}(\varphi)=\int f(z, \zeta) \varphi(x) d x$ is continuous in $(y, \zeta) \in C_{\delta} \times \mathscr{K}$.

$\underline{A}$. It may happen that the limit value of $F_{y, \zeta}(\varphi)$ as $y \rightarrow 0$ in $C_{\delta}$ exists so that $F_{y, \zeta}(\varphi)$ can be extended to a function defined in $\left(C_{\delta} \cup\{0\}\right) \times \mathscr{K}$ and continuous in every $\bar{C}_{\delta}^{\prime} \times \mathscr{K}$.

If this holds for every $\varphi \in \mathscr{D}(\Omega)$ the limit $F_{\zeta}=F_{0, \zeta}$ defines a continuous function from $\zeta$ into $\mathscr{D}^{\prime}(\Omega)$.

A necessary and sufficient condition for this is:

B. For every compact subset $K \subset \Omega$ and compact subcone $\bar{C}^{\prime}$ of $C$ we can find $\delta>0, M>0$, and non-negative integer $m$, such that

$$
|f(z, \zeta)| \leqq M|y|^{-m}, \quad(z, \zeta) \in\left(K+i\left[\bar{C}_{\delta}^{\prime}-\{0\}\right]\right) \times \mathscr{K} .
$$

Here $\delta$ may depend on $K$, and $M$ and $m$ on $K$ and $\bar{C}^{\prime}$.

3. Before we proceed to the somewhat lengthy proof of Theorem 1 we indicate in the form of corollaries some special cases and simple consequences of the theorem.

Cor. 1. This is the case when $f(z, \zeta)$ is independent of $\zeta$ and gives the criterion for existence of the boundary value $F$ of a function $f(z)$ holomorphic in $O_{C}(\Omega)$ (II. 2).

Cor. 2. Here we are concerned with the existence of the boundary value $F_{\mathscr{C}}$ of $f(z)$ on a $C$-like analytic curve $\mathscr{C}$.

If we apply Theorem 1 , with $z=\tau$ and $\mathscr{K}=A\left(\bar{C}_{\delta}^{\prime}, \varepsilon, k\right)$, to $g(\tau, \zeta)$ $=f(\alpha(\tau)+\zeta)$ we get the criterion formulated in (II. 5). (Observe that $C$ etc. in (II. 5) does not correspond to $C$ etc. in Theorem 1.) 
Cor. 3. Assume $f(z)$ is holomorphic in $O_{C}(\Omega)$ and has a boundary value on some $\Sigma \subset \Omega$ containing a $C$-like analytic curve $\mathscr{C}$. Given $\varphi \in \mathscr{D}(I)$, let $I^{\prime}$ be a relatively compact open subinterval of $I$ containing supp $\varphi$, and $\varepsilon>0$ be the distance from the image of $I^{\prime}$ on $\mathscr{C}$ to the boundary of $\Sigma$. Then

$$
F_{\xi}(\varphi)=\lim \int f(\alpha(t+i s)+\xi+i \eta) \varphi(i) d t, \quad s \rightarrow+0, \quad \eta \rightarrow 0 \text { in } C,
$$

exists and is an infinitely differentiable function of $\xi$ for $|\xi|<\varepsilon$, where $\partial^{p} / \partial \xi^{p} F_{\xi}(\varphi)=\lim \int f^{(p)}(\alpha(t+i s)+\xi+i \eta) \varphi(t) d t, s \rightarrow+0, \eta \rightarrow 0$ in $C$.

The value of $\partial^{p} / \partial \xi^{p} F_{\xi}(\varphi)$ for $\xi=0$ exists for every $\varphi \in \mathscr{D}(I)$ and defines a distribution in $\mathscr{D}^{\prime}(I)$ which is just the boundary value of $f^{(p)}(z)$ on $\mathscr{C}$. Hence $f(z)$ has boundary values of infinite order on $\mathscr{C}$.

If the distance $d$ betueen $\mathscr{C}$ and the boundary of $\Sigma i$ s positive the above construction defines a set $\left\{F_{\xi} ;|\xi|<d\right\}$ of distributions in $\mathscr{D}^{\prime}(I)$, infinitely differentiable in $\xi$.

Proof of Cor. 3. Given $\varphi \in \mathscr{D}(I)$ and corresponding $\varepsilon$. For every positive $\varepsilon^{\prime}<\varepsilon$ the set $K^{\prime}=$ closure of $\bigcup_{t \in I}\left\{x ;|x-\alpha(t)| \leqq \varepsilon^{\prime}\right\}$ is compact in $\Sigma$.

As $f(z)$ has boundary value on $\Sigma$, Cor. 1 shows that for every $\bar{C}^{\prime}$ there are $M$ and $m$ such that

$$
|f(z)| \leqq M|y|^{-m}, \quad z \in K^{\prime}+i\left(\bar{C}_{\delta}^{\prime}-\{0\}\right) .
$$

Put $z=\alpha(\tau)+\xi+i \eta$. If $t$ belongs to $L$, compact subset of $I^{\prime}$, and $s>0$ is small enough we certainly have $|y|>M_{1} s$ with some $M_{1}>0$, so $|f(\alpha(\tau)+\xi+i \eta)| \leqq M^{\prime} s^{-m}, \quad \tau \in L+i\left(0, \delta^{\prime}\right), \quad|\xi| \leqq \varepsilon^{\prime}, \quad \eta \in \bar{C}_{\delta}^{\prime}$. Application of Theorem 1 (cf. Cor. 2) shows the existence of $F_{\zeta}$ as element in $\mathscr{D}^{\prime}\left(I^{\prime}\right)$, continuous in $\zeta$ for $|\xi| \leqq \varepsilon^{\prime}, \eta \in \bar{C}_{\delta}^{\prime}$. For $\eta=0$ we obtain the desired $F_{\xi}(\varphi)$ which is of course continuous in $\xi$.

To prove that $F_{\xi}(\varphi)$ is infinitely differentiable we note that for $\eta \neq 0 F_{\xi+i \eta}(\varphi)$ is evidently infinitely differentiable, as we may then without hesitation differentiate under the integral sign. As the derivative $f^{(p)}(z)$ has boundary value on $\Sigma$ (because $f(z)$ has, ef. remark in (II. 3)) we find that $\lim \partial^{p} / \partial \xi^{p} F_{\xi+i \eta}(\varphi), \eta \rightarrow 0$, exists as a continuous function which is approached uniformly in $\xi$ when $\eta \rightarrow 0$. This implies that $F_{\xi}(\varphi)$ is infinitely differentiable.

The proof of the remaining statements in Cor. 3 should be obvious from the proof already given.

Cor. 4. Assume $f_{ \pm}(z)$ are holomorphic in $O_{ \pm C}(\Omega)$, respectively, and have boundary values $F_{ \pm}$on $\Sigma$ as $y \rightarrow 0$ in $C$ and $-C$.

If $F_{+}=F_{-}$, then for every $\varphi \in \mathscr{D}(I)$ the functions $F_{ \pm \xi}(\varphi)$ defined in Cor. 3 are equal. Especially this implies that the boundary values of $f_{ \pm}(z)$ on $\mathscr{C}$ are equal to infinite order.

Proof of Cor. 4. For $\eta \neq 0$

$$
F_{\xi+i \eta}(\varphi)=\int f(\alpha(t)+\xi+i \eta) \varphi(t) d t
$$


and as $\eta \rightarrow 0$

uniformly for $|\xi|<\varepsilon$.

$$
F_{\xi}(\varphi)=\lim F_{\xi+i \eta}(\varphi)
$$

Taking a $C^{\infty}$ function $\psi(\xi)$ with supp $\psi<\{\xi ;|\xi|<\varepsilon\}$ we get

$$
\begin{aligned}
\int F_{\xi}(\varphi) \psi(\xi) d \xi & =\lim \int f(\alpha(t)+\xi+i \eta) \varphi(t) \psi(\xi) d t d \xi(\eta \rightarrow 0) \\
& =\lim \int f(x+i \eta) \chi(x) d x(\eta \rightarrow 0),
\end{aligned}
$$

where we have introduced coordinates $(x=\alpha(t)+\xi, t)$ instead of $(\xi, t)$, and

Hence we find

$$
\chi(x)=\int \varphi(t) \psi(x-\alpha(t)) d t \in \mathscr{D}(\Sigma)
$$

$$
\int F_{+\xi}(\varphi) \psi(\xi) d \xi=F_{+}(\chi)=F_{-}(\chi)=\int F_{-\xi}(\varphi) \psi(\xi) d \xi .
$$

As $F_{ \pm \xi}(\varphi)$ are continuous in $\xi$ and $\psi$ is arbitrary, we must have $F_{+\xi}(\varphi)$ $=F_{-\xi}(\varphi)$.

4. Proof of Theorem 1. For shortness we shall in the following in general suppress the dependence on the variable $\zeta$, varying in $\mathscr{K}$, as its inclusion is in most cases trivial. This depends on the fact that all functions we consider will turn out to be continuous and, $\mathscr{K}$ being compact, even uniformly continuous in $\zeta$. E.g. it is easily seen from the Cauchy formula that as we have assumed $f(z, \zeta)$ continuous simultaneously in $z$ and $\zeta$, then so is any $z$-derivative $f^{(p)}(z, \zeta)$.

We first formulate a seemingly more special theorem which can be shown to imply Theorem 1.

Theorem 1'. Assume $f(z)$ holomorphic in some $O_{C}(\Omega)$ where the closure $\bar{\Gamma}$ of the product of the positive half-axis $\Gamma=\left\{y ; y_{i}>0\right\}$ is a compact subcone of $C$, i.e. $\bar{\Gamma}-\{0\} \subset C$.

Then the following statements are equivalent:

a. $\lim \int f(z) \varphi(x) d x, y \rightarrow 0$ in $\bar{\Gamma}_{\delta}-\{0\}$, exists for every $\varphi \in \mathscr{D}(\Omega)$, defining a distribution $F \in \mathscr{D}^{\prime}(\Omega)$.

b. For every compact set $K$ in $\Omega$ we can find $\delta>0, M>0$, and a set $\left(m_{1}, \ldots, m_{n}\right)$ of non-negative integers such that

$$
|f(z)| \leqq M y_{1}^{-m_{1}} \ldots y_{n}^{-m_{n}}, \quad z \in K+i \Gamma_{\delta} .
$$

We first show that Theorem 1' implies Theorem 1.

Let $C$ be the convex cone of Theorem 1. Every compact subcone $\bar{C}^{\prime}$ can be covered by a finite number of convex polygonal cones $\bar{C}_{p}^{\prime}=\left\{y=\Sigma v_{i} y^{\prime(i)}, \quad v_{i}>0 ; y^{\prime(i)}, \quad i=1, \ldots, n\right.$, linearly independent vectors $\}$ compact in $C$. Every $\bar{C}_{p}^{\prime}$ can be somewhat enlarged to $\bar{C}_{p}=\left\{y=\Sigma v_{i} y^{(i)}\right\}$, so that $\bar{C}_{p}^{\prime}$ is compact in $\bar{C}_{p}$ and $\bar{C}_{p}$ is compact in $C$.

The inverse of the transformation $x=\Sigma w_{i} y^{(i)}$ then maps $\Omega+i \bar{C}_{p}$ one-to-one on some $\Omega^{\prime}+i \bar{\Gamma}$. It is easy to see that Theorem $1, \underline{A}$ (in $z$ ) implies Theorem $1^{\prime}, \underline{a}$ (in $w$ ), and Theorem $1^{\prime}, \underline{b}$ (in $w$ ) implies Theorem 1 , 
$\underline{B}$ (in $z$, and with subcone $\bar{C}_{p}^{\prime}, m=m_{1}+\cdots+m_{n}$ ). As $\bar{C}^{\prime}$ is covered by a finite number of $\bar{C}_{p}^{\prime}$ we have shown that provided Theorem $1^{\prime}$ is true, statement $\underline{A}$ implies statement $\underline{B}$ in Theorem 1 .

The inverse implication follows in a similar way by mapping $\Omega+i \bar{C}_{p}^{\prime}$ on some $\Omega^{\prime \prime}+i \Gamma$.

We now proceed to the proof of Theorem $1^{\prime}$.

First we note that if $f(z)$ satisfies $\underline{a}$. then so does the primitive

$$
f^{(-1,0 . .)}\left(z, z^{0}\right)=\int_{z_{1}^{0}}^{z_{1}} f\left(z_{1}^{\prime}, z_{2}, \ldots, z_{n}\right) d z_{1}^{\prime} .
$$

Here $z^{0}$ is a fixed point in $O_{\Gamma}(C)$.

In fact, writing

$$
\begin{aligned}
f^{(-1, .)}\left(z, z^{0}\right)=f^{(-1, .)}\left(z^{1}, z^{0}\right) & +\int_{x_{1}^{1}}^{x_{1}} f\left(x_{1}^{\prime}+i y_{1}^{1}, z_{2}, \ldots, z_{n}\right) d x_{1}^{\prime} \\
& +i \int_{y_{1}^{1}}^{y_{1}} f\left(x_{1}+i y_{1}^{\prime}, z_{2}, \ldots, z_{n}\right) d y_{1}^{\prime},
\end{aligned}
$$

where $z^{1}=\left(z_{1}^{1}, z_{2}, \ldots, z_{n}\right)$ is some point in $K+i\left(\bar{\Gamma}_{\delta}-\{0\}\right)$, we see that when $y \rightarrow 0$ in $\bar{\Gamma}$ the first two terms remain in the domain of holomorphy of $f(z)$ and thus make no difficulty. For the third term we get after averageing over $\varphi(x)$ the expression

$$
\int_{y_{1}^{1}}^{y_{1}} d y_{1}^{\prime}\left(\int f\left(x_{1}+i y_{1}^{\prime}, z_{2}, \ldots, z_{n}\right) \varphi(x) d x\right)
$$

and here the inner integral is continuous in $\left(y_{1}^{\prime}, y_{2}, \ldots, y_{n}\right)$ up to the boundary so that the outer integral converges as $y_{1} \rightarrow 0$.

It follows by induction that if $\underline{\alpha}$. is true for $f(z)$ it is true also for any iterated primitive $f^{(-p)}\left(z, z^{0}\right)$, where $p=\left(p_{1}, \ldots, p_{n}\right), p_{i}$ being nonnegative integers.

Conversely, as derivation is a continuous operation in $\mathscr{D}^{\prime}(\Omega)$ it follows that if $f(z)$ satisfies $\underline{a}$. then so does any derivative $f^{(p)}(z)$.

This last observation is used to prove $\underline{b} . \Rightarrow \underline{a}$. We assume $\underline{b}$. to hold and form the $p=\left(m_{1}+1, \ldots, m_{n}+1\right)$ times iterated primitive $f(-p)\left(z, z^{0}\right)$. This function is easily realized to be continuous in $K+i \bar{\Gamma}_{\delta}$, and then certainly satisfies $\underline{a}$. This is then also true for $f(z)=D^{p} f^{(-p)}\left(z, z^{0}\right)$.

We now go over to the more difficult proof of the implication $\underline{a} . \Rightarrow \underline{b}$. First we note that every compact $K$ in $\Omega$ can be covered by a finite number of open polyintervals (sets of the form $I_{1} \times \ldots \times I_{n}$, where $I_{i}$ is a bounded interval), relatively compact in $\Omega$ and containing closed polyintervals also covering $K$. Each $\varphi \in \mathscr{D}(\Omega)$ with support in $K$ can then be written as a finite sum of test functions, each having its support in 
one of these open polyintervals ([12], Ch. I, Th. II). We can then without loss of generality assume that our $\Omega$ is an open bounded polyinterval and that $f(z)$ is holomorphic in every point of $\Omega+i\left(\bar{\Gamma}_{\delta}-\{0\}\right)$ for some $\delta>0$.

For simplicity of notation we carry through the proof for $n=2$, putting $f=f(z, w), w=u+i v, \Omega=I_{1} \times I_{2}$. The case $n>2$ is proved similarly, and the proof for the considerably simpler case $n=1$ is also easily deduced from the proof given here.

We thus assume that $f(z, w)$ satisfies $\underline{a}$. If we take a sequence of rectangles $K \subset \Omega_{K} \subset \bar{\Omega}_{K} \subset \Omega$, where $\Omega_{K}=I_{1}^{\prime} \times I_{2}^{\prime}$, then $f(z, w)$ and its distribution limit as $(y, v) \rightarrow 0$ can for test functions in $\mathscr{D}\left(\Omega_{K}\right)$ be represented in the form ([12], Ch. TII, remark following Th. XXIII) $f(z, w)=D_{x}^{p} D_{u}^{q} F(x, u ; y, v)$, where $F(x, u ; y, v)$ is continuous in $(z, w) \in \Omega_{K}+i[0, \delta / \sqrt{2}) \times[0, \delta / \sqrt{2})$. [ . .) here denotes an interval including the left end-point. If we take into account the dependence on $\zeta$, $F$ should be denoted $F(x, u ; y, v, \zeta)$ and is simultaneously continuous in all variables.

Integrating $p$ times in $x$ we find

$$
f^{(-p, 0)}(z, w)=D_{u}^{q} F(x, u ; y, v)+\sum_{\nu=0}^{p-1} x^{v} a_{\nu}(u ; y, v),
$$

where $a_{v}(u ; y, v)$ are distributions in $\mathscr{D}^{\prime}\left(I_{2}^{\prime}\right)$, depending on the parameters $y$ and $v$.

It is not hard to realize that we can find $p$ functions

$\varphi_{\nu} \in \mathscr{D}\left(I_{1}^{\prime}\right), \quad v=0,1, \ldots, p-1, \quad$ such that $\int x^{\nu} \varphi_{\mu}(x) d x=\delta_{\mu \nu}$.

We then get

$$
a_{v}(u ; y, v)=\int f^{(-n, 0)}(z, w) \varphi_{\nu}(x) d x-\int D_{u}^{q} F(x, u ; y, v) \varphi_{\nu}(x) d x .
$$

This relation shows that $a_{v}(u ; y, v)$ is, as distribution in $u$, continuous in $(y, v) \in[0, \delta / \sqrt{2}) \times\left[0, \delta / \sqrt{2)}\right.$. We conclude that $\int f^{(-p, 0)}(z, w) \psi(u) d u$ is continuous in $z \in I_{1}^{\prime}+i[0, \delta / \sqrt{2}), v \in[0, \delta / \sqrt{2})$, for every $\psi \in \mathscr{D}\left(I_{2}^{\prime}\right)$. The same thing is true for $\int f^{(-p,-q)}(z, w) \psi(u) d u$, as follows from an argument analogous to the one used just in the beginning of the proof.

We now form

$$
\begin{aligned}
f^{(-p,-g)}(z, w)=F(x, u ; y, v) & +\sum_{\nu=0}^{p-1} x^{v} b_{\nu}(u ; y, v) \\
& +\sum_{\mu=0}^{q-1} u^{\mu} c_{\mu}(x ; y, v) .
\end{aligned}
$$

With $\psi_{\nu} \in \mathscr{D}\left(I_{2}^{\prime}\right), v=0,1, \ldots q-1$, satisfying $\int u^{v} \psi_{\mu}(u) d u=\delta_{\mu \nu}$ we rewrite

$$
\begin{aligned}
& b_{\nu}(u ; y, v)=\bar{b}_{\nu}(u ; y, v)+\sum_{\mu=0}^{q-1} u^{\mu} \int b_{\nu}\left(u^{\prime} ; y, v\right) \psi_{\mu}\left(u^{\prime}\right) d u^{\prime} \\
& c_{v}(x ; y, v)=\bar{c}_{v}(x ; y, v)+\sum_{\mu=0}^{p-1} x^{\mu} \int c_{v}\left(x^{\prime} ; y, v\right) \varphi_{\mu}\left(x^{\prime}\right) d x^{\prime}
\end{aligned}
$$


Then $\left\{\bar{b}_{\nu}\right\}$ and $\left\{\bar{c}_{\nu}\right\}$ are zero on all $\left\{\psi_{\nu}\right\}$ and $\left\{\varphi_{\nu}\right\}$, respectively. Therefore we can write

$$
\begin{aligned}
f^{(-p,-q)}(z, w)= & F(x, u ; y, v)+\sum x^{\nu} \bar{b}_{\nu}(u ; y, v) \\
& +\sum u^{\mu} \bar{c}_{\mu}(x ; y, v)+\sum x^{\nu} u^{\mu} d_{\nu \mu}(y, v) .
\end{aligned}
$$

Applying this distribution equation on $\varphi_{\nu}(x) \psi_{\mu}(u)$ we find that $d_{\nu \mu}(y, v)$ are continuous in $y, v$, and from

$$
\begin{aligned}
\bar{c}_{\nu}(x ; y, v)= & \int f^{(-p,-q)}(z, w) \psi_{\nu}(u) d u-\int F(x, u ; y, v) \psi_{v}(u) d u \\
& -\sum_{\mu} x^{\mu} d_{\mu \nu}(y, v)
\end{aligned}
$$

we see that $\bar{c}_{v}(x ; y, v)$ can be assumed to be continuous in $x, y$, and $v$. The analogous conclusion holds for $\bar{b}_{v}(u ; y, v)$. Then $f^{(-p,-q)}(z, w)$ can be continued to a function continuous in $\Omega_{K}+i \bar{\Gamma}_{\delta}$.

The Cauchy integral

$$
f(z, w)=\frac{p ! q !}{(2 \pi i)^{2}} \iint \frac{f^{\left(-p_{0}-q\right)}\left(z^{\prime}, w^{\prime}\right)}{\left(z^{\prime}-z\right)^{p+1}\left(w^{\prime}-w\right)^{q+1}} d z^{\prime} d w^{\prime}
$$

where part of the integration includes the real boundary sides of $K$, finally gives the majoration

$$
|f(z, w)| \leqq M y^{-p-1} v^{-q-1}, \quad(z, w) \in K+i \Gamma_{\delta}^{\prime}, \quad \text { some } \delta^{\prime}>0 .
$$

The proof of Theorem $\mathrm{l}^{\prime}$ is thus complete.

5. Remark to Theorem 1. Theorem $1^{\prime}, \underline{b}$. is essentially the criterion given by Borchers ([6], Lemma 10) for the existence of boundary value of a function holomorphic in the product of the upper half planes. Theorem $\mathrm{I}^{\prime}, \underline{b}$. is a stronger restriction on $f(z)$ than Theorem $1, \underline{B}$, applied to $C=\Gamma$.

In fact, one can show by a proof somewhat more involved than the proof of Theorem $1^{\prime}$ that for a function $f(z)$ holomorphic in some $O_{\Gamma}(\Omega)$ the bound $1^{\prime}, \underline{b}$. is equivalent to the following statement.

For every $\varphi \in \mathscr{D}(\Omega), F_{y}(\varphi)=\int f(z) \varphi(x) d x$ can be continued to a function continuous in $y$ in some $\bar{\Gamma}_{\delta}$. Thus the boundary value exists not only as $y \rightarrow 0$ in $\Gamma$, but also for $y$ on some part of the boundary of $\Gamma$ near $y=0$.

6. Theorem 2 (Uniqueness theorem). If $f(z)$ is holomorphic in $O_{C}(\Omega)$ and if for $f(z)$ and every derivative $f^{(p)}(z)$

$\lim \int f^{(p)}(\alpha(t+i s)) \varphi(t) d t=0, s \rightarrow 0$, for every $\varphi \in \mathscr{D}(I)$, then $f(z)=0$ throughout $O_{C}(\Omega)$.

Proof. If we put $g(\tau)=f^{(p)}(\alpha(\tau))$ and choose a test function $\beta(t)$ with support in $\{t ;|t|<\varepsilon\}$, the regularized

$$
g_{\beta}(\tau)=\int g\left(t^{\prime}+i s\right) \beta\left(t-t^{\prime}\right) d t^{\prime}
$$


is holomorphic in $\left\{\tau ; \varepsilon<t<1-\varepsilon, 0<s<\delta^{\prime}\right\}$, and the limit value 0 as $s \rightarrow 0$, is approached uniformly in $t$. Schwarz' reflection principle then implies $g_{\beta}(\tau) \equiv 0$, and taking a sequence of test functions approaching $\delta(t)$ we find $g(\tau) \equiv 0$. Thus $f(z)$ and every derivative is equal to zero at some point in $O_{C}(\Omega)$, so we conclude $f(z) \equiv 0$.

Remark. Theorem 2 combined with Theorem 1, Cor. 3, implies that if $f(z)$ is holomorphic in $O_{C}(\Omega)$ and has boundary value zero on some $\Sigma$, then $f(z) \equiv 0$ (cf. [1], Th. $2-17$, where this result is a corollary of the ordinary edge of the wedge theorem).

7. We now begin with the proof of the main theorem. For clarity we give several theorems of increasing generality: Theorem 3 deals with the case where $C$ contains $\bar{\Gamma}$ as a compact subcone and $\mathscr{C}$ is a line segment, in Theorem 4 we generalize to an arbitrary convex cone $C$, and finally in Theorem $5 \mathscr{C}$ is allowed to be an arbitrary $C$-like analytic curve.

Theorem 3. Assume $f_{ \pm}(z)$ holomorphic in $O_{ \pm C}(\Omega)$, respectively, where $C>\bar{\Gamma}-\{0\} . \mathscr{C}$ is the segment $\left\{x=x^{0}+\alpha t, 0<t<1, \alpha=\left(\alpha_{1}, \ldots, \alpha_{n}\right)\right.$, $\left.\alpha_{i}>0\right\}$, and the open polyinterval $R=I_{1} \times \ldots \times I_{n}, I_{v}=\left\{x_{v} ; x_{v}^{0}<x_{v}<x_{v}^{0}\right.$ $\left.+\alpha_{v}\right\}$, lies in $\Omega$.

We furthermore assume that $f_{ \pm}(z)$ have equal boundary values of infinite order on $\mathscr{C}$ when $s \rightarrow \pm 0, \zeta \rightarrow 0$ in $A\left( \pm \bar{\Gamma}_{\delta}, \varepsilon, k\right)$, some $k>0$ (cf. (II. $\left.\tilde{5}\right)$ ).

Then there is a common analytic continuation $f(z)$ of $f_{ \pm}(z)$ which is holomorphic in some open neighbourhood of $R$ in $C^{n}$. This neighbourhood depends on $O_{ \pm C}(\Omega)$ and $\mathscr{C}$, but not on $f_{ \pm}(z)$.

Proof. We first prove Theorem 3 under the further restriction that the boundary values of $f_{ \pm}(z)$ and all derivatives are $C^{\infty}$ functions in $t$, which are approached uniformly in $t$ in every compact subset of $\mathscr{C}$.

By a change of scale we can assume $R$ to be a polysquare, i.e. having all sides equal. Evidently the conclusion of Theorem 3 is that the interior of every compact sub-polysquare $\bar{R}^{\prime}$ of $R$ having the same centre as $R$ and sides parallell to those of $R$ is in the holomorphy domain of a continuation $f(z)$.

In order to prove this we take a fixed $\bar{R}^{\prime}$. As $\bar{R}^{\prime}$ is compact in $\Omega$ there exists an $\varepsilon>0$, such that for every $x^{\prime} \in \bar{R}^{\prime}$ the sets $\left\{z ;\left|x_{i}-x_{i}^{\prime}\right| \leqq \varepsilon\right.$, $\left.0< \pm y_{i} \leqq \varepsilon, i=1, \ldots, n\right\}$ are in $O_{ \pm C}(\Omega)$, respectively. Choose a positive $x<1$. Then we will show that there is a $\delta>0$ (depending on $\varepsilon$ and $x$ ) with the property: if $R_{\delta}$ is a polysquare in $\bar{R}^{\prime}$, sides $=\delta$ and parallell to those of $\bar{R}^{\prime}$, such that the boundary values of $f_{ \pm}(z)$ exist and are equal to infinite order on the diagonal of $R_{\delta}$ (including the end points), then the interior of $R_{x \delta}$ is in the holomorphy domain of a continuation. Here $R_{x \delta}$ is a polysquare concentric to $R_{\delta}$, sides $=\varkappa \delta$.

Suppose we have shown the existence of a $\delta$ with these properties. Then we can start from the diagonal of $\bar{R}^{\prime}$, where we know that the boundary values exist and are equal, and fill up this diagonal with an 
infinite number of $R_{\delta}: s$. In the real part of the region of holomorphy thus obtained we take a new diagonal parallell to the previous one, and successively repeat the procedure. For the simplest case $n=2$, where $\bar{R}^{\prime}$ is a square, sides equal to $d$, it is not hard to see that we can carry out the extension in $N=d / \delta$ steps. After these $N$ steps a square, concentric with $\bar{R}^{\prime}$ and with sides equal to $d-N \delta(1-x)=d x$, is in the holomorph domain. Evidently the whole interior of $\bar{R}^{\prime}$ can be covered if such a $\delta$ exists for every $x<1$. The same conclusion is easily realized to be valid also for $n>2$.

Now we prove the existence of the above $\delta$. By translation and rescaling we can achieve that $R_{\delta}$ is the square

the sets

$$
\left\{x ;-1<x_{i}<1, \quad i=1, \ldots, n\right\} ;
$$

$$
\left\{z ;\left|x_{i}\right| \leqq 2 \varepsilon / \delta, \quad 0< \pm y_{i} \leqq 2 \varepsilon / \delta\right\}
$$

are in the holomorphy domains of $f_{ \pm}(z)$, respectively. On the diagonal of $R_{\delta}$ (including the end points) the limit values of $f_{ \pm}(z)$ and all derivatives exist as $C^{\infty}$ functions and are equal in pairs. We keep the original notations $f_{ \pm}(z)$, though actually we should use new symbols for the functions obtained after the variable transformations.

Study the function

$$
\begin{aligned}
f\left(z_{1}, \ldots, z_{n}\right) & =\frac{1}{2 \pi i} \int_{C_{+}} \frac{f_{+}\left(\frac{u+z_{1}}{1+u z_{1}}, \ldots \frac{u+z_{n}}{1+u z_{n}}\right)}{u} d u \\
& +\frac{1}{2 \pi i} \int_{C_{-}} \frac{t_{-}\left(\frac{u+z_{1}}{1+u z_{1}}, \ldots \frac{u+z_{n}}{1+u z_{n}}\right)}{u} d u
\end{aligned}
$$

$C_{+}$and $C_{-}$are here the upper and lower halves of the unit circle $|u|=1$ in the complex $u$-plane.

For $|u|=1$ we find (here $z=x+i y$ is one-dimensional!)

$$
\frac{u+z}{1+u z}=\frac{2 x+\left(1+|z|^{2}\right) \operatorname{Re} u}{|1+u z|^{2}}+i \frac{\left(1-\mid z i^{2}\right) \operatorname{Im} u}{|1+u z|^{2}} \text {. }
$$

If $|z| \leqq x<1$ we thus have

$$
\left|\operatorname{Re}\left(\frac{u+z}{1+u z}\right)\right| \leqq \frac{4}{(1-x)^{2}}, \quad\left|\operatorname{Im}\left(\frac{u+z}{1+u z}\right)\right| \leqq \frac{1}{(1-x)^{2}} .
$$

Choosing $2 \varepsilon / \delta>\frac{4}{(1-x)^{2}}$, i.e. $\delta<\varepsilon(1-x)^{2} / 2$, we find that the arguments of the functions $f_{ \pm}$in the integrals above are inside $O_{ \pm C}(\Omega)$ along $C_{ \pm}$, except at the points $u= \pm 1$, if $\left|z_{i}\right| \leqq \varkappa, i=1, \ldots, n$.

When $u$ approaches \pm 1 the arguments of $f_{ \pm}$approach the end points of the diagonal of $R_{\delta}$. To ensure that the arguments $\zeta$ (cf. the statement of Theorem 3$)$ go to zero in $A\left( \pm \bar{\Gamma}_{\delta}, \varepsilon, k\right)$ for the given $k$ we have to 
restrict $z_{i}$ further, so that the condition $|\xi|<k|\eta|$ is fulfilled. A closer investigation shows that we must impose the condition $4 \Sigma y_{i}^{2}$ $<k^{2} \Sigma\left(1-\left|z_{i}\right|^{2}\right)^{2}$. Denote by $O_{x}$ the set of $z$-values satisfying this condition and the condition $\left|z_{i}\right|<\varkappa, i=1, \ldots, n . O_{x}$ is an open set, the real section of which is $R_{* \delta}$. It is now rather easy to verify that $f\left(z_{1}, \ldots, z_{n}\right)$ is holomorphic for $z \in O_{x}$. To show that $f$ is holomorphic in each $z_{i}$ we have to differentiate under the integral signs. As $f_{ \pm}$and their derivatives approach the limit values uniformly in $A\left( \pm \bar{\Gamma}_{\delta}, \varepsilon, k\right)$, the critical points $u= \pm 1$ do not give any trouble.

It remains to identify $f\left(z_{1}, \ldots, z_{n}\right)$ with $f_{ \pm}(z)$ in $O_{ \pm C}(\Omega) \cap O_{x}$. $f\left(z_{1}, \ldots, z_{n}\right)$ is then the desired continuation, holomorphic at every point of $R_{x \delta}$.

Computing the derivative $D^{p}\left(=D_{z_{1}}^{p_{1}} \ldots D_{z_{n}}^{p_{n}}\right)$ of $f\left(z_{1}, \ldots\right)$ and putting $z_{1}=\cdots z_{n}=t$, we find

$$
\begin{aligned}
D^{p} f(t, \ldots, t) & =\frac{1}{2 \pi i} \int_{C_{+}} \frac{d u}{u}\left[\frac{\left(1-u^{2}\right)^{p}}{(1+u t)^{2}}\left(D^{p} f_{+}\right)\left(\frac{u+t}{1+u t}, \ldots\right)+0(u)\right] \\
& +\frac{1}{2 \pi i} \int_{C_{-}} \cdots . \quad|p|=p_{1}+\cdots+p_{n} .
\end{aligned}
$$

$0(u)$ here denotes terms containing lower order derivatives of $f_{+}$and vanishing for $u=0$.

For fixed $t$ in $(-x, x)$ the function in [] and the corresponding function in the second integral are holomorphic in $|u| \leqq 1, \operatorname{Im} u>0$ and $\operatorname{Im} u<0$, respectively, and their limit values which are continuous and uniformly approached on the real $u$-axis are equal. According to the classical Painlevé's theorem ([1], p. 74) they form a single holomorphic function in $|u| \leqq 1$, and the two integrals in the formula for $D^{p} f(t, \ldots, t)$ can be added to give a single closed contour integral. Applying Cauchy's formula we get the value of the function in [] for $u=0$, i.e.

$$
D^{p} f(t, \ldots, t)=D^{p} f_{ \pm}(t, \ldots, t) .
$$

Application of Theorem 2 (really, as the limits are continuous we can apply Schwarz' reflection principle directly) gives

$$
f\left(z_{1}, \ldots, z_{n}\right)=f_{ \pm}\left(z_{1}, \ldots, z_{n}\right) \quad \text { in } \quad O_{ \pm C}(\Omega) \cap O_{\varkappa} .
$$

$O_{\varkappa}$ may depend of the particular functions $f_{ \pm}(z)$ through the value of $k$. But as we have found that the boundary value of $f_{ \pm}(z)$ is actually a function holomorphic in some complex neighbourhood of $R_{\varkappa \delta}$, it is no longer necessary to keep the restriction $|\xi|<k|\eta|$. Thus $f\left(z_{1}, \ldots, z_{n}\right)$ is holomorphic in $\left\{z ;\left|z_{i}\right|<x\right\}$, and it is then realized that the construction of the domain of holomorphy of the "global" $f(z)$ depends only on $O_{ \pm C}(\Omega)$ and $\mathscr{C}$, but not on $f_{ \pm}(z)$. 
We have now proved Theorem 3 for the case of $C^{\infty}$ boundary values.

The general case with distribution valued boundary values is reduced to this case by regularization. With some small $\varepsilon>0$ take $\beta \in \mathscr{D}((-\varepsilon, \varepsilon))$ and form $f_{ \pm}^{\beta}(z)=\int f(z+\alpha t) \beta(t) d t$. This function then fulfills the assumption for the case with $C^{\infty}$ boundary values, if the region are appropriately reduced to take care of the smearing out over $(-\varepsilon, \varepsilon)$. We indicate these new regions by an index $\varepsilon$. Applying the earlier result we then find that there exists an analytic continuation $f^{\beta}(z)$ holomorphic in some complex neighbourhood of $R_{\varepsilon}$. Therefore for every $\beta$

$$
\lim \int f_{ \pm}(\zeta+\alpha(t+i s)) \beta(t) d t, \quad s \rightarrow \pm 0, \quad \text { exist for } \zeta \in \bar{R}_{\varepsilon} \pm i \bar{\Gamma}_{\delta} .
$$

According to Theorem 1 this implies the restriction

$$
\left|f_{+}(\zeta+\alpha \tau)\right| \leqq M s^{-m}, \quad 0<s \leqq \delta^{\prime}, \quad \zeta \in \bar{R}_{\varepsilon}+i \bar{\Gamma}_{\delta} .
$$

Given $z=x+i y=\zeta+\alpha \tau$, i.e. $y_{i}=\eta_{i}+\alpha_{i} s$, we evidently for $y \in \Gamma_{\delta}$ get $\eta \in \bar{\Gamma}_{\delta}$ if we choose $s=\min y_{i} / \alpha_{i}$. Then we find

$$
s>\Pi \frac{y_{i}}{\alpha_{i}+y_{i}} \text {, and }\left|f_{+}(z)\right| \leqq M\left[\Pi\left(\alpha_{i}+y_{i}\right)^{m}\right] \cdot y_{1}^{-m} \ldots y_{n}^{-m} .
$$

Thus, according to Theorem $I^{\prime}$ (III. 4) $f_{+}(z)$ (and similarly $f_{-}(z)$ ) has a boundary value on $R_{\varepsilon}$ as $y \rightarrow 0$ in $\bar{\Gamma}_{\delta}-\{0\}$. As $\varepsilon$ can be taken arbitrarily small, $f_{ \pm}(z)$ have boundary values $F_{ \pm}$on $R$.

These boundary values are easily seen to be equal. With $\varphi \in \mathscr{D}\left(R_{\varepsilon}\right)$ we get

$$
\begin{aligned}
\int f^{\beta}(x) \varphi(x) d x & =\lim \int f_{ \pm}(x+i y)\left[\int \beta(t) \varphi(x-\alpha t) d t\right] d x \\
& =F_{ \pm}(\beta * \varphi) .
\end{aligned}
$$

But the set $\{\psi=\beta * \varphi\}$ is dense in $\mathscr{D}\left(R_{\varepsilon}\right)($ let $\beta(t)$ approach $\delta(t))$ so $F_{+}=F_{-}$on $\mathscr{D}\left(R_{\varepsilon}\right)$ and, as $\varepsilon$ is arbitrary, on $\mathscr{D}(R)$.

To verify that this boundary distribution is actually a real-analytic function $f(x)$ we have to check that for every test function $\gamma$ with arbitrarily small support, supp $\gamma \subset\{x ;|x|<\varepsilon\}$ the regularized $f^{\gamma}(x)$ $=\int f\left(x-x^{\prime}\right) \gamma\left(x^{\prime}\right) d x^{\prime}$ is analytic ([12], Ch. VI, Th. XXIV). But $f^{\gamma}(x)$ $=\lim f_{ \pm}^{\nu}(z), y \rightarrow 0$ in $\pm \bar{\Gamma}_{\delta}$, where $f_{ \pm}^{\gamma}(z)=\int f_{ \pm}\left(z-x^{\prime}\right) \gamma\left(x^{\prime}\right) d x$, and $f_{ \pm}^{\prime}(z)$ are easily seen to satisfy the conditions of Theorem 3 , with $C^{\infty}$ boundary values equal on $\mathscr{C}_{\varepsilon}$. Hence $f_{ \pm}^{\prime}(z)$ have a common analytic continuation. The restriction of this continuation to $y=0$ must be just $f^{\gamma}(x)$.

Thus the boundary distribution is a real-analytic function $f(x)$ which can be continued to some complex neighbourhood of $R$. By Theorem 2 this complex extension must be an analytic continuation of $f_{ \pm}(z)$. This then shows that the limit values on $\mathscr{C}$ are actually $C^{\infty}$ functions, uniformly approached, so using the special case of Theorem 3 again we get a continuation $f(z)$ of $f_{ \pm}(z)$ to a neighbourhood of $R$, which depends only 14 Commun. math. Phys., Vol. 8 
on the geometry of the problem, not on the special functions $f_{ \pm}(z)$. This last step is necessary since the original extension of the real-analytic distribution $f(x)$ to complex $z$ may well give a holomorphy domain depending on $f(x)$.

8. Theorem 4. Assume $f_{ \pm}(z)$ holomorphic in $O_{ \pm C}(\Omega)$, respectively, and

We assume that

$$
\mathscr{C}=\left\{x=x^{0}+\alpha t, \quad 0<t<1, \quad \alpha \in C\right\} .
$$

$$
B=\left(x^{0}+C\right) \cap\left(x^{0}+\alpha-C\right) \subset \Omega .
$$

If $f_{ \pm}(z)$ have equal boundary values of infinite order on $\mathscr{C}$ there is a common analytic continuation $f(z)$ of $f_{ \pm}(z)$, holomorphic in some complex neighbourhood of $B$. This neighbourhood depends on $O_{ \pm C}(\Omega)$ and $\mathscr{C}$ but not on $f_{ \pm}(z)$.

Proof. With $C$ and $B$ as in the assumptions and $x$ a point in $B$ we can always find a convex polygonal cone $C^{\prime}$, such that $\bar{C}^{\prime}$ is a compact subcone of $C$, and $x \in\left(x^{0}+C^{\prime}\right) \cap\left(x^{0}+\alpha-C^{\prime}\right)$. In fact,

means

$$
x \in\left(x^{0}+C\right) \cap\left(x^{0}+\alpha-C\right)
$$

$$
x=x^{0}+\alpha^{\prime}, \quad \alpha^{\prime} \in C \quad \text { and } \quad x=x^{0}+\alpha-\alpha^{\prime \prime}, \quad \alpha^{\prime \prime} \in C .
$$

It is easy to see that we can choose a convex polygonal cone $C^{\prime}$, relatively compact in $C$ and containing $\alpha, \alpha^{\prime}$, and $\alpha^{\prime \prime}$. This cone then fulfills the conditions.

We now make a linear transformation of $C^{\prime}$ onto $\Gamma$. The image of the point $x$ in $B$ will then belong to the $\Gamma$-convex hull of the image of $\mathscr{C}$. The assumptions of Theorem 3 are fulfilled, and transforming back we find that $x$ is in the domain of holomorphy of a continuation $f(z)$ of $f_{ \pm}(z)$.

9. Theorem 5. Assume $f_{ \pm}(z)$ holomorphic in $O_{ \pm C}(\Omega)$, respectively, and $\mathscr{C}$ some $C$-like analytic curve in $\Omega . B(\mathscr{C}, \Omega, C)$ denotes the $C$-convex hull of $\mathscr{C}$ in $\Omega$ (II. 9 ).

If $f_{ \pm}(z)$ are equal to infinite order on $\mathscr{C}$ there is a common analytic continuation $f(z)$ of $f_{ \pm}(z)$, holomorphic in some complex neighbourhood of $B(\mathscr{C}, \Omega, C)$. This neighbourhood depends on $O_{ \pm C}(\Omega)$ and $B$ but not on $f_{ \pm}(z)$.

Proof. We first show that to every point $x \in \mathscr{C}$ we can find an open neighbourhood $N(x)$, independent of $f_{ \pm}(z)$, in $C^{n}$, such that there is a continuation of $f_{ \pm}(z)$ in $N(x)$.

Let $x\left(t_{0}\right)$ be a point of $\mathscr{C}$. As $x^{\prime}\left(t_{0}\right) \in C$ at least one $x_{i}^{\prime}\left(t_{0}\right)$ is different from zero, say $x_{1}^{\prime}\left(t_{0}\right)$. Then $z_{1}=z_{1}(\tau)$ is invertible in some complex neighbourhood of $t_{0}$, giving $\tau=t_{0}+\gamma\left(z_{1}\right)$ where $\gamma$ is holomorphic in some neighbourhood of $x_{1}\left(t_{0}\right)$ in the $z_{1}$-plane. Introducing new coordinates $\zeta_{1}=\gamma\left(z_{1}\right), \zeta_{i}=z_{i}-z_{i}\left(t_{0}+\gamma\left(z_{1}\right)\right), i=2, \ldots, n$, we get a mapping $\zeta(z)$, holomorphic in both directions, of some neighbourhood $N_{1}$ of $x\left(t_{0}\right)$ onto 
some neighbourhood $N_{2}$ of the origin in $\zeta$-space. If $N_{1}$ is chosen small enough it is clear from continuity that we can find a cone $C_{2}$ in $\zeta$-space, such that the image of $N_{1} \cap\left(R^{n} \pm i C\right)$ contains $N_{2} \cap\left(R^{n} \pm i C_{2}\right)$ and that the image of $N_{1} \cap \mathscr{C}$, which is a part of the line $\zeta_{2}=\cdots=\zeta_{n}=0$, is $C_{2}$-like in $N_{2} \cap\left(R^{n} \pm i\{0\}\right) \cdot g_{ \pm}(\zeta)=f_{ \pm}(z(\zeta))$ are then holomorphic in $N_{2} \cap\left(R^{n} \pm i C_{2}\right)$, and when $\operatorname{Im} \zeta$ goes to zero in $\pm C_{2} g$ are easily seen to fulfill the criterion in Theorem 1, Cor. 2, and have thus equal boundary values of infinite order on the image of $N_{1} \cap \mathscr{C}$. According to Theorem 4 $g_{ \pm}(\zeta)$ have a common continuation $g(\zeta)$ holomorphic in some neighbourhood of the origin. Transforming back to the $z$-coordinates we get a continuation $f(z)$ of $f_{ \pm}(z)$ where $f(z)$ is holomorphic in some neighbourhood of $x\left(t_{0}\right)$.

Repeating this procedure we get a continuation of $f_{ \pm}(z)$ to a neighbourhood $\cup\{N(x) ; x \in \mathscr{C}\}$ of $\mathscr{C}$. On $C$-like straight segments in the real part of this neighbourhood we can apply Theorem 4, and continuing this process we finally get a function $f(z)$ holomorphic in some complex neighbourhood of $B(\mathscr{C}, \Omega, C)$, That this neighbourhood is independent of $f_{ \pm}(z)$ is obvious, and a moment's reflection shows that it must also be independent of the special $\mathscr{C}$ generating $B(\mathscr{C}, \Omega, C)$.

Acknowledgement. One of the authors ( $\AA$. K.) would like to express his gratitude to the Swedish Council for Nuclear Research for financial support during part of the work.

\section{References}

1. Streater, R. F., and A. Wightman: PCT, spin and statistics and all that. New York: Benjamin 1964.

2. Vladinirov, V. S.: Metody teorii funktsii mnogikh complexnykh peremennykh. Moscow: Izd. Nauka 1964.

3. Epstein, H.: J. Math. Phys. 1, 524 (1960).

4. Borchers, H. J.: Nuovo Cimento 19, 787 (1961).

5. Trtchmarsh, E. C.: Introduction to the theory of Fourier integrals. Second edition. Oxford: Clarendon Press 1948.

6. Borchers, H. J.: Nuovo Cimento 33, 1600 (1964).

7. Trummann, H.-G.: Math. Z. 59, 61 (1953).

8. - Math. Z. 77, 106 (1961).

9. Bremermann, H.: Distributions, complex variables, and Fourier transforms. Berkeley: Addison-Wesley 1965.

10. Gremenberg, O. W.: J. Math. Phys. 3, 859 (1962).

11. Wightman, A.: Analytic functions of several complex variables. In: DE Witt, C., and R. Onnes: Relations de dispersion et particules élémentaires. Les Houches: Hermann 1960.

12. Schwartz, L.: Théorie des distributions. Paris: Hermann 1950 or 1966.

Dr. A. Kolm and Dr. B. Nagel

Department of Theoretical Physics Royal Institute of Technology Stockholm 70 (Sweden) 